\title{
Dynamics and Simulation of the Effects of Wind on UAVs and Airborne Wind Measurement*
}

\author{
Hyoung Sik CHOI, ${ }^{1)}$ Sangjong LeE, ${ }^{2)}$ Hyeok RYU, ${ }^{1)}$ Hyunchul SHIM, ${ }^{3)}$ and Cheolkeun $\mathrm{HA}^{4)}$ \\ ${ }^{1)}$ Korea Aerospace Research Institute, Daejeon 305-806, Republic of Korea \\ ${ }^{2)}$ Department of Aircraft Mechanical Engineering, Inha Technical College, Incheon 402-752, Republic of Korea \\ ${ }^{3)}$ Department of Aerospace Engineering, Korea Advanced Institute of Science and Technology, Daejeon 305-701, Republic of Korea \\ ${ }^{4)}$ Department of Aerospace Engineering, University of Ulsan, Ulsan 680-749, Republic of Korea
}

\begin{abstract}
This paper presents a simulation of wind effects on a flying unmanned aerial vehicle (UAV) and a simulation of wind measurements taken from an airborne UAV. The wind acts as a disturbance on the flying UAV by altering its attitude and velocity. Therefore, the airborne measurement should be taken dynamically to reflect the instantaneous motion of a vehicle that is affected by wind. In order to understand and validate the algorithm that measures wind and the interaction between wind and the UAV, an investigation using simulation is extremely valuable. Considering real applications, this study implements modeling of the interaction between wind and a UAV from the perspective of the UAV's onboard sensors, and a wind measurement formula is clarified based on the established model. The results of the simulation confirm that, if an accurate measurements for angle-of-attack and sideslip are available, the wind can be accurately measured regardless of the control scheme used in flight.
\end{abstract}

Key Words: Airborne, Wind Effect, Wind Measurement, Simulation, UAV

\section{Nomenclature}

$\alpha, \beta$ : angles of attack and sideslip

$\phi, \theta$ : roll and pitch angles

$u, v, w: x, y$, and $z$ velocity in body axis

$p, q, r$ : roll, pitch, and yaw rates

$U_{a}$ : airspeed measured from probe

$V_{T}$ : total airspeed (including wind speed)

$V_{N}, V_{E}$ : north and east ground speed components

$V_{g}:$ ground speed

$W_{N, E, D}^{\text {from }}$ : wind from north, east, and down

$W_{N, E, D}^{\text {to }}$ : wind toward north, east, and down

$\psi$ : yaw angle

$\psi^{\prime}$ : course angle, $\tan ^{-1}\left(V_{E} / V_{N}\right)$

$\gamma$ : flight path angle

$m$ : aircraft mass

$g$ : gravity acceleration parameter

$h$ : altitude

$P_{N}$ : aircraft position in north axis

$\dot{P}_{N}$ : ground velocity to north, $\dot{P}_{N}=V_{N}$

$\boldsymbol{P}$ : aircraft position vector

$\boldsymbol{v}$ : aircraft moving velocity vector

I: aircraft moment of inertia matrix

$\boldsymbol{F}, \boldsymbol{M}$ : force and moment in body axis

$\omega$ : body angular rate vector

$\boldsymbol{\Omega}$ : angular rate cross product, $\boldsymbol{\Omega}=(\boldsymbol{\omega} \times)$

$\boldsymbol{\Phi}$ : Euler angle vector

Subscripts
$a$ : body axis (including wind)
$b$ : body axis

(C) 2015 The Japan Society for Aeronautical and Space Sciences

*Received 29 July 2013; accepted for publication 23 October 2013.
$N, E, D$ : earth axis (north, east, down)

\section{Introduction}

Because the wind acts as a disturbance on UAVs, it is necessary to include wind effects and investigate the controller's performance under windy conditions during the controller design and hardware-in-the-loop (HIL) test phase. ${ }^{1,2)}$ Furthermore, recent advances in the utilization of wind as a useful energy (e.g., soaring flight for low-energy consumption), ${ }^{3)}$ not just a disturbance, demand more extensive studies of airborne wind estimation and measurement. The airborne methods extract a wind component from data collected by the UAV's onboard sensors (e.g., air-data system (ADS), global positioning system (GPS), and inertial navigation system (INS)) in real time.

The difference between estimation and measurement is whether or not angle-of-attack $(\alpha)$ and sideslip $(\beta)$ vane sensors are utilized. This study refers to measurement as the method that utilizes $\alpha$ and $\beta$ sensors ${ }^{4-7)}$ and estimation as the method that does not. ${ }^{8)}$

Langelaan et al. elaborated on the formulation of measurement (direct computation of the wind field) and estimation (computing wind from vehicle response). ${ }^{6}$

The overall objective of this study is to implement simulations, validate the equations that are proposed in literature mentioned in the references, ${ }^{4-7)}$ and verify their measurement or estimation results. As these tasks require a thorough understanding of the dynamics of the interaction between the wind and the UAV, this study starts by modeling the interaction from an onboard sensor's perspective and clarifying the wind measurement formula. Next, a flight simulation program of the UAV under windy conditions is established, 
Table 1. Required onboard sensor outputs.

\begin{tabular}{cll}
\hline \multicolumn{1}{c}{ Sensor } & \multicolumn{1}{c}{ Output } & \multicolumn{1}{c}{ Description } \\
\hline \multirow{2}{*}{ ADS } & $\alpha, \beta$ & Angle of attack, sideslip \\
& $V_{T}, h$ & Total airspeed, pressure altitude \\
\hline \multirow{2}{*}{ GPS*/INS } & $P_{N}, P_{E}, P_{D}$ & Ground position \\
& $V_{N}, V_{E}, V_{D}$ & Ground speed component \\
\hline \multirow{2}{*}{ GPS/INS* } & $A_{x}, A_{y}, A_{z}$ & Body acceleration \\
& $p, q, r$ & Body angular rate \\
\hline \multirow{2}{*}{ GPS/INS* } & $u, v, w$ & Body speed component \\
& $\phi, \theta, \psi$ & Euler angles \\
\hline
\end{tabular}

and the outputs from the sensors are determined. These outputs form the basis of the wind measurement.

Finally, the established simulation is validated with measurement cases in which wind is present.

The sensors considered in the study are ADS and GPS/ INS. The GPS/INS is assumed to yield an output that is perfectly identical to the output from the simulation.

The information output from the simulation sensors is shown in Table 1 , where ${ }^{*}$ indicates the dominant sensor of the GPS/INS system. The outputs specified in Table 1 are used for feedback to the UAV controller as well as measurement of the wind.

The ADS provides measurements for the angle-of-attack $(\alpha)$, sideslip $(\beta)$ and flight speed, while the GPS provides position and time derivatives of position. The INS basically provides acceleration, angular velocity, and as a result of integration with the GPS, highly accurate Euler attitude angles.

The UAV used for the simulation in this study utilizes ambient speed $\left(U_{a} \cong V_{T}\right)$ for speed control, pressure altitude $\left(h=-P_{D}\right)$ for altitude control, and attitude angles and rates $(\phi, \theta$ and $p, q, r)$ for internal attitude control. For heading control, two different control schemes are simulated. One utilizes the yaw angle $(\psi)$ feedback, where yaw angle denotes the direction in which the vehicle's nose is pointing, and the other one utilizes the course angle $\left(\psi^{\prime}\right)$ feedback, where the course angle denotes the direction of the horizontal flight path. The yaw angle deviates from the course angle in the presence of a side wind.

\section{Wind Effect on Aircraft Motion}

This chapter presents mathematical modeling of the correlation between the wind and UAV movement from a sensor's perspective. The subscripts indicate the sensors to which the quantity corresponds. $A D S$ and INS are the corresponding sensors, and the wind is applied wind.

\subsection{Wind effect}

In general, as the Dryden model demonstrates, wind models can be constructed using the velocity component and angular rate component of the wind. When the velocity component of the wind is applied to the motion of a UAV, the component can be defined as Eq. (1) with respect to the body coordination system. In Eq. (1), the + wind is defined as wind that approaches the UAV. The angular rate of the wind is directly added to $p, q$ and $r$, as shown in Fig. 2 .
For simplicity of analysis, this study considers only the velocity component of the wind.

$$
\left[\begin{array}{c}
u_{a} \\
v_{a} \\
w_{a}
\end{array}\right]_{A D S}=\left[\begin{array}{c}
u \\
v \\
w
\end{array}\right]_{I N S}+\left[\begin{array}{c}
u \\
v \\
w
\end{array}\right]_{\text {wind }}
$$

\subsection{Equations of motion}

The equations of motion of the vehicle are formulated as follows:

Force Equation $\left(\dot{\boldsymbol{v}}_{b}=-\boldsymbol{\Omega}_{b} \boldsymbol{v}_{b}+\boldsymbol{T}_{b / e} g+\boldsymbol{F}_{b} / m\right)$ :

$$
\begin{aligned}
{\left[\begin{array}{c}
\dot{u} \\
\dot{v} \\
\dot{w}
\end{array}\right]_{I N S}=\frac{\boldsymbol{F}_{b}}{m}+\boldsymbol{T}_{b / e}\left[\begin{array}{c}
0 \\
0 \\
g
\end{array}\right]_{I N S} } \\
-\left[\begin{array}{rrr}
0 & -r & q \\
r & 0 & -p \\
-q & p & 0
\end{array}\right]_{I N S}\left[\begin{array}{c}
u \\
v \\
w
\end{array}\right]_{I N S}
\end{aligned}
$$

Moment Equation $\left(\boldsymbol{I} \dot{\boldsymbol{\omega}}_{b}=-\boldsymbol{\Omega}_{b} \boldsymbol{I} \boldsymbol{\omega}_{b}+\boldsymbol{M}_{b}\right)$ :

$$
\left[\begin{array}{c}
\dot{p} \\
\dot{q} \\
\dot{r}
\end{array}\right]_{I N S}=\boldsymbol{I}^{-1}\left(\boldsymbol{M}_{b}-\left[\begin{array}{c}
p \\
q \\
r
\end{array}\right]_{I N S} \times \boldsymbol{I}\left[\begin{array}{l}
p \\
q \\
r
\end{array}\right]_{I N S}\right)
$$

Kinematic Equation $\left(\dot{\boldsymbol{\Phi}}=E(\boldsymbol{\Phi}) \boldsymbol{\omega}_{b}\right)$ :

$$
\left[\begin{array}{c}
\dot{\phi} \\
\dot{\theta} \\
\dot{\psi}
\end{array}\right]_{I N S}=\left[\begin{array}{ccc}
1 & \tan \theta \sin \phi & \tan \theta \cos \phi \\
0 & \cos \phi & -\sin \phi \\
0 & \sin \phi / \cos \theta & \cos \phi / \cos \theta
\end{array}\right]_{I N S}\left[\begin{array}{c}
p \\
q \\
r
\end{array}\right]_{I N S}
$$

Navigation Equation $\left(\dot{\boldsymbol{P}}_{N, E, D}=\boldsymbol{T}_{b / e^{-1}} \boldsymbol{v}_{b}\right)$ :

$$
\left[\begin{array}{c}
\dot{P}_{N} \\
\dot{P}_{E} \\
\dot{P}_{D}
\end{array}\right]_{I N S}=\boldsymbol{T}_{b / e}{ }^{-1}\left[\begin{array}{c}
u \\
v \\
w
\end{array}\right]_{I N S}
$$

where the earth-to-body transformation matrix is given below:

$$
\begin{aligned}
\boldsymbol{T}_{b / e}= & {\left[\begin{array}{ccc}
1 & 0 & 0 \\
0 & \cos \phi & \sin \phi \\
0 & -\sin \phi & \cos \phi
\end{array}\right]\left[\begin{array}{ccc}
\cos \theta & 0 & -\sin \theta \\
0 & 1 & 0 \\
\sin \theta & 0 & \cos \theta
\end{array}\right] } \\
& \cdot\left[\begin{array}{ccc}
\cos \psi & \sin \psi & 0 \\
-\sin \psi & \cos \psi & 0 \\
0 & 0 & 1
\end{array}\right]
\end{aligned}
$$

\section{Wind Measurement}

\subsection{Equation}

From Eq. (1), the wind measurement is formulated as Eq. (6). It is apparent from the equation that wind measurement is possible if the precise values of the INS velocity component and the ADS velocity component (including wind effect) are available. 


$$
\left[\begin{array}{c}
u \\
v \\
w
\end{array}\right]_{\text {wind }}=\left[\begin{array}{c}
u_{a} \\
v_{a} \\
w_{a}
\end{array}\right]_{A D S}-\left[\begin{array}{c}
u \\
v \\
w
\end{array}\right]_{I N S}
$$

The velocity component of the INS is derived from Eq. (5) as Eq. (7). The subscript GPS is used because integrating GPS and INS results in approximately the same ground speed for INS as in GPS.

$$
\left[\begin{array}{c}
u \\
v \\
w
\end{array}\right]_{I N S}=\boldsymbol{T}_{b / e}\left[\begin{array}{c}
\dot{P}_{N} \\
\dot{P}_{E} \\
\dot{P}_{D}
\end{array}\right]_{G P S} \cong \boldsymbol{T}_{b / e}\left[\begin{array}{c}
\dot{P}_{N} \\
\dot{P}_{E} \\
\dot{P}_{D}
\end{array}\right]_{I N S}
$$

where $\dot{\boldsymbol{P}}_{I N S} \cong \dot{\boldsymbol{P}}_{G P S}=\left[V_{N}, V_{E}, V_{D}\right]_{G P S}^{\mathrm{T}}$

The velocity component of the ADS is calculated using total airspeed, $\alpha$ and $\beta$, as shown in Eq. (8). ${ }^{4-7)}$ It is clear from this equation that the accuracy of the calculated ADS velocity component depends on the accuracies of $\alpha, \beta$ and $V_{T}$.

$$
\left[\begin{array}{c}
u_{a} \\
v_{a} \\
w_{a}
\end{array}\right]_{A D S}=\frac{V_{T}}{\sqrt{1+\tan ^{2} \alpha+\tan ^{2}} \beta}\left[\begin{array}{c}
1 \\
\tan \beta \\
\tan \alpha
\end{array}\right]_{A D S}
$$

As a result, the wind measurement formula with respect to the body coordinate system becomes Eq. (9), where + indicates incoming wind.

$$
\left[\begin{array}{c}
u \\
v \\
w
\end{array}\right]_{\text {wind }}=\frac{V_{T}}{D}\left[\begin{array}{c}
1 \\
\tan \beta \\
\tan \alpha
\end{array}\right]_{A D S}-\boldsymbol{T}_{b / e}\left[\begin{array}{c}
\dot{P}_{N} \\
\dot{P}_{E} \\
\dot{P}_{D}
\end{array}\right]_{G P S}
$$

where, $D=\sqrt{1+\tan ^{2} \alpha+\tan ^{2} \beta}$.

The wind measurement, explained so far in respect to the body reference frame, is converted to the ground reference frame, as shown in Eq. (10). $+W_{N}^{\text {from }}$ indicates the wind coming from the north.

$$
\left[\begin{array}{c}
W_{N}^{\text {from }} \\
W_{E}^{\text {from }} \\
W_{D}^{\text {from }}
\end{array}\right]_{\text {wind }}=\left[\boldsymbol{T}_{b / e^{-1}}\right]_{I N S}\left[\begin{array}{c}
u_{a} \\
v_{a} \\
w_{a}
\end{array}\right]_{A D S}-\left[\begin{array}{c}
\dot{P}_{N} \\
\dot{P}_{E} \\
\dot{P}_{D}
\end{array}\right]_{G P S}
$$

Equation (10) is expanded into Eqs. (11)-(13).

$$
\begin{aligned}
W_{N}^{\text {from }}= & \frac{V_{T}}{D}[\cos \theta \cos \psi \\
& +\tan \beta(\sin \phi \sin \theta \cos \psi-\cos \phi \sin \psi) \\
& +\tan \alpha(\cos \phi \sin \theta \cos \psi+\sin \phi \sin \psi)]_{a d s} \\
& -\left(\dot{P}_{N}\right)_{G P S} \\
W_{E}^{\text {from }}= & \frac{V_{T}}{D}[\cos \theta \sin \psi \\
& +\tan \beta(\sin \phi \sin \theta \sin \psi+\cos \phi \cos \psi) \\
& +\tan \alpha(\cos \phi \sin \theta \sin \psi-\sin \phi \cos \psi)]_{a d s} \\
& -\left(\dot{P}_{E}\right)_{G P S} \\
W_{D}^{\text {from }}= & \frac{V_{T}}{D}[-\sin \theta+\tan \beta(\sin \phi \cos \theta) \\
& +\tan \alpha(\cos \phi \cos \theta)]_{a d s}-\left(\dot{P}_{D}\right)_{G P S}
\end{aligned}
$$

\begin{tabular}{|c|c|c|c|}
\hline Ref. & \multicolumn{3}{|c|}{ Equations in references (symbol replaced) } \\
\hline Ref. 4), p. 4 & \multicolumn{3}{|c|}{$\begin{aligned} W_{D}^{\text {from }}= & -\dot{P}_{D}+\frac{V_{T}}{D}[-\sin \theta+\tan \beta(\sin \phi \cos \theta) \\
& +\tan \alpha(\cos \phi \cos \theta)]\end{aligned}$} \\
\hline $\begin{array}{l}\text { Ref. 5), Eq. (3) } \\
\text { Ref. 6), Eq. (22) }\end{array}$ & $\left.\begin{array}{l}W_{N}^{t o} \\
W_{E}^{t o} \\
W_{D}^{t o}\end{array}\right]=$ & $\left.\begin{array}{l}\dot{P}_{N} \\
\dot{P}_{E} \\
\dot{P}_{D}\end{array}\right]-\left[\boldsymbol{T}_{b / e}{ }^{-1}\right]$ & {$\left[\begin{array}{c}u \\
v \\
w\end{array}\right]$} \\
\hline Ref. 7), Eq. (5) & \multicolumn{3}{|c|}{$\begin{aligned} W_{D}^{t o}= & \dot{P}_{D}-\frac{V_{T}}{D}[-\sin \theta+\tan \beta(\sin \phi \cos \theta) \\
& +\tan \alpha(\cos \phi \cos \theta)]\end{aligned}$} \\
\hline
\end{tabular}

Table 2. Equation verification.

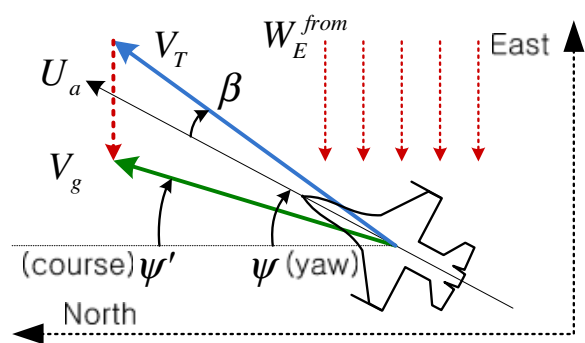

Fig. 1. Heading angle and speed definition.

\subsection{Verification}

This section verifies previously-derived wind measurement equations via comparison with other reference equations.

Table 2 shows reference equations described in the terms used in this paper. All of the equations, from literature in the references, ${ }^{4-7)}$ correspond exactly to the results of this study.

\section{Simulation}

This section evaluates the impact of the wind on a UAV and the results of the simulation with regard to airspeed, altitude and heading hold control. The simulated UAV has a cruise speed of $60 \mathrm{~km} / \mathrm{h}$ and is equipped with a proportional-integral-derivative (PID) controller. ${ }^{9)}$ The applied control law is summarized as follows.

Altitude, speed, and heading are controlled in terms of pitch angle, throttle and roll angle, respectively. The feedback state is formulated as explained in Section 1. $U_{a}$, the approximation of total airspeed $V_{T}$, is used in airspeed control, and the feedback heading can be selected from yaw angle and course angle.

The speed and heading angles are defined in Fig. 1. The ground speed $V_{g}$ is equal to $V_{T}$ less the wind velocity (i.e., $\overrightarrow{\boldsymbol{V}}_{G P S}=\overrightarrow{\boldsymbol{V}}_{A D S}+\overrightarrow{\boldsymbol{V}}_{\text {wind }}$ ). This corresponds to the results of Eq. (10).

Probe airspeed $U_{a}$ is measured through a pitot probe aligned with the $x$-axis in the body reference frame.

\subsection{Interaction between wind and UAV}

The architecture of the simulation program is shown in Fig. 2. The influence of the wind on the UAV is explained with the block diagram sequence as follows:

1. Wind blows (uvw_wind)

2. $\alpha, \beta$ and airspeed $V_{T}$ change (ADS)

3. Aerodynamic force changes (aerodynamics) 


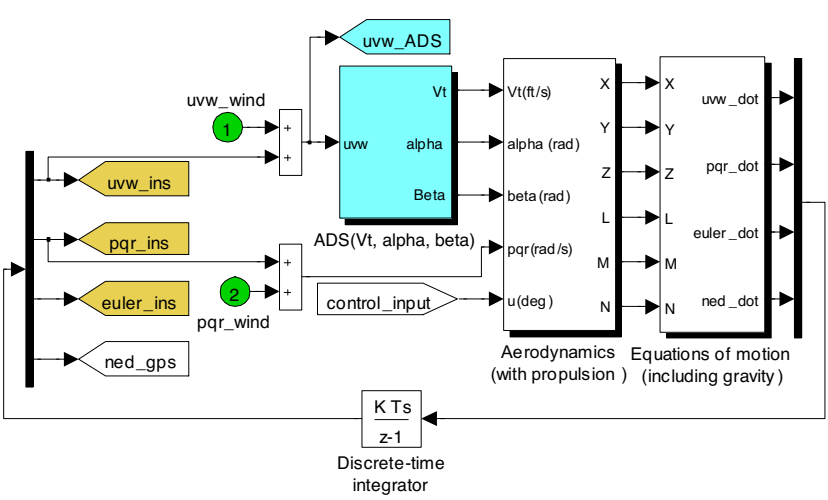

Fig. 2. Nonlinear UAV simulation program with wind included.

4. The changed force acts on the UAV's mass and inertia (EoM)

5. The motion of the UAV changes (integrator)

This sequence of influences is conducted in one time step in the iterative simulation, and the effect of the wind on the changed motion of the UAV is applied in the next time step.

It is assumed in this study that $\alpha$ and $\beta$ are ideally measured without lever arms, as Eq. (14) shows.

$$
\alpha_{A D S}=\tan ^{-1} \frac{w_{a}}{u_{a}}, \quad \beta_{A D S}=\tan ^{-1} \frac{v_{a}}{u_{a}}
$$

In order to obtain the accurate total airspeed $V_{T}$, the inlet of the probe needs to be oriented in the direction of the wind. However, as the probe is generally fixed on the vehicle body, this study employs the following ideal assumption:
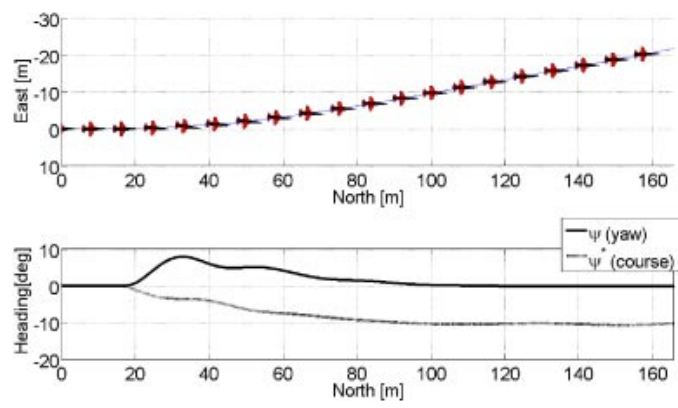

Fig. 3. Trajectory and heading (case 1).
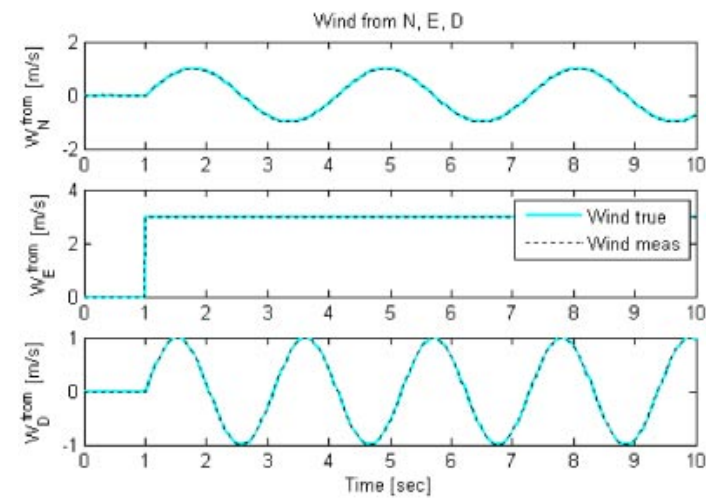

Fig. 5. Applied wind and measurement (case 1).

$$
V_{T} / D \cong U_{a}=u_{I N S}+u_{\text {wind }}
$$

The wind interaction and results of the simulation are discussed for the following two cases, which are differentiated by heading angle selection:

Case 1: yaw angle $(\psi)$ feedback

Case 2: course angle $\left(\psi^{\prime}\right)$ feedback

Figures 3 and 4 present the horizontal trajectory and heading response. Figures 5 and 6 present applied wind and measurement results for both cases. As Fig. 3 show, the yaw angle control maintains its heading but yields a shifted horizontal trajectory due to the effect of the wind. The course angle control, shown in Fig. 4, yields a very small trajectory deviation. In this case, the yaw angle turns toward the wind direction. The results verify that the implemented simulation performs correctly. Furthermore, Figs. 5 and 6 indicate that the wind is accurately measured regardless of the control laws.

\subsection{Wind effect on UAVs}

Figures 7-10 show the flight parameters and control characteristics that represent the effects of wind on the UAV.

Figures 7 and 8 present the performance of the UAV controller. As the figures show, heading hold is successfully performed in both cases under the same altitude, speed and wind conditions.

Figures 9 and 10 show the behaviors of $\alpha, \beta$ and flight path angle in the presence of wind. Although the assumption of the leftmost column in Table 3 holds if there is no wind, Figs. 9 and 10 indicate that this assumption is not valid under windy conditions.

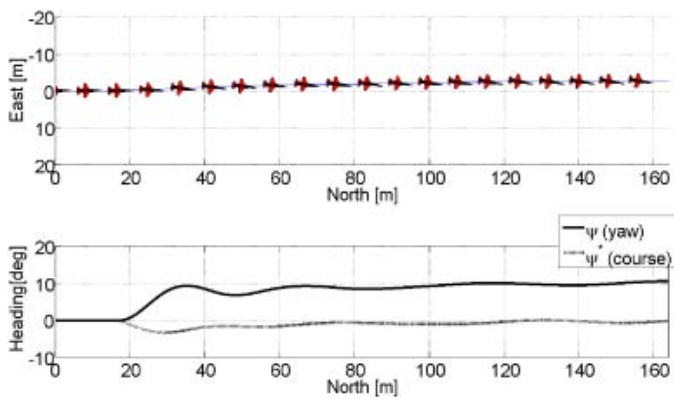

Fig. 4. Trajectory and heading (case 2).
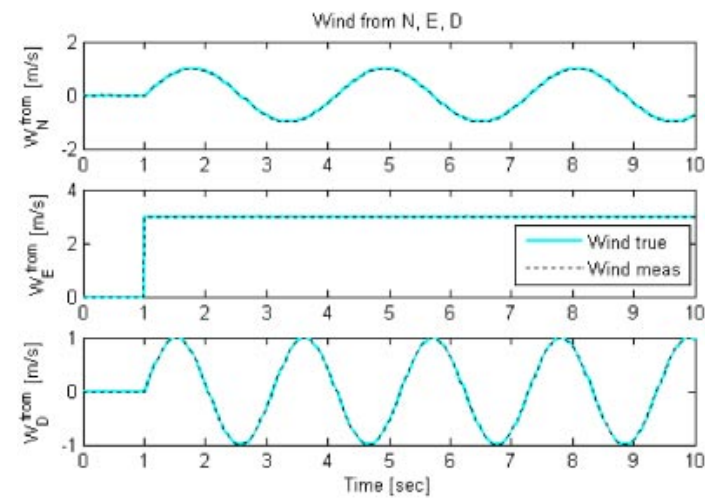

Fig. 6. Applied wind and measurement (case 2). 

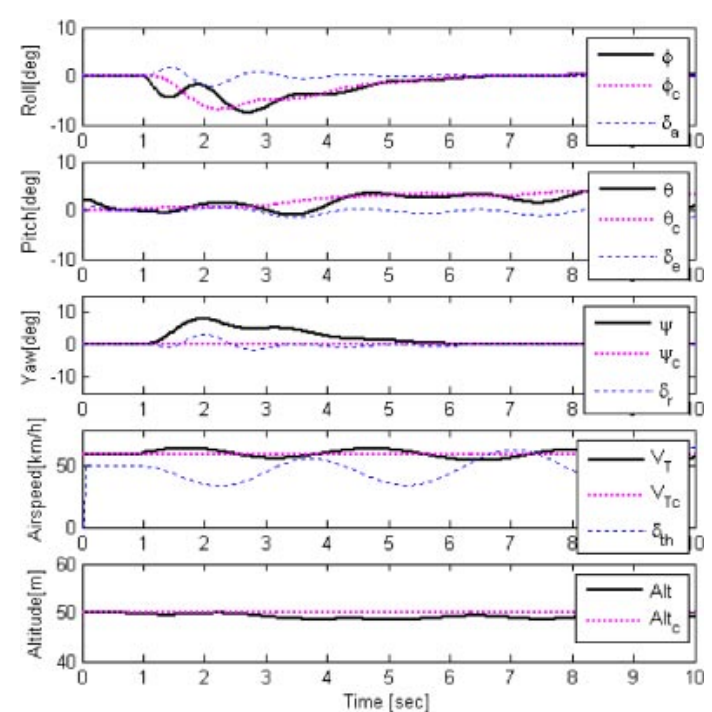

Fig. 7. Autopilot characteristics (case 1).
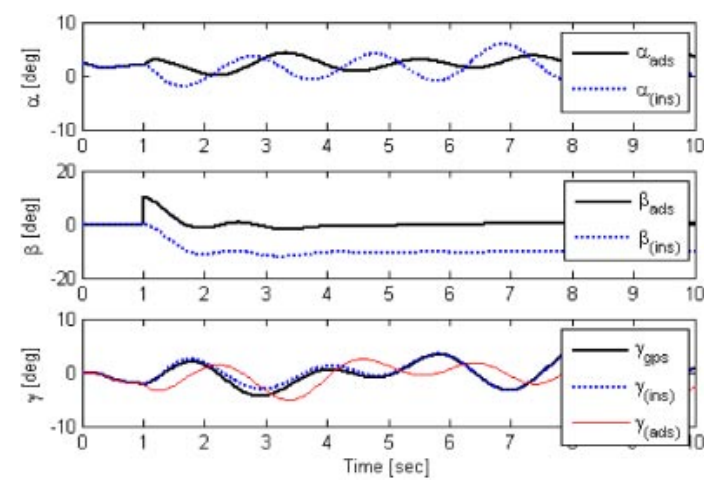

Fig. 9. Flight parameters (case 1).

On the other hand, under windy conditions, INS yields an approximation of $\gamma$ that is much closer to the GPS $\gamma$ value than the ADS approximation is. The GPS yields highly accurate $\gamma$ values $\left(\gamma_{G P S}=\tan ^{-1}\left(-V_{D} / \sqrt{V_{N}^{2}+V_{E}^{2}}\right)\right.$.

In Figs. 9 and 10, it is notable that the measured values of $\beta$ ( $\left.\beta_{A D S}\right)$ converge to 0 for both cases.

\section{Conclusion}

This study conducted modeling of the interaction between wind and aircraft from the onboard sensor's perspective, and it simplified wind measurement formulas based on the model. In addition, a program for simulating UAV flight under windy conditions was implemented. The outputs of the simulation program were determined to represent the sensor's perspective, and the method for measuring the wind utilized these outputs.

The program was verified using simulation case studies that applied windy conditions to different heading control schemes. When yaw angle control was used, the simulation captured a flight path shift due to side wind. Meanwhile, the course angle control accurately captured the change in heading due to the side wind. These results indicate that
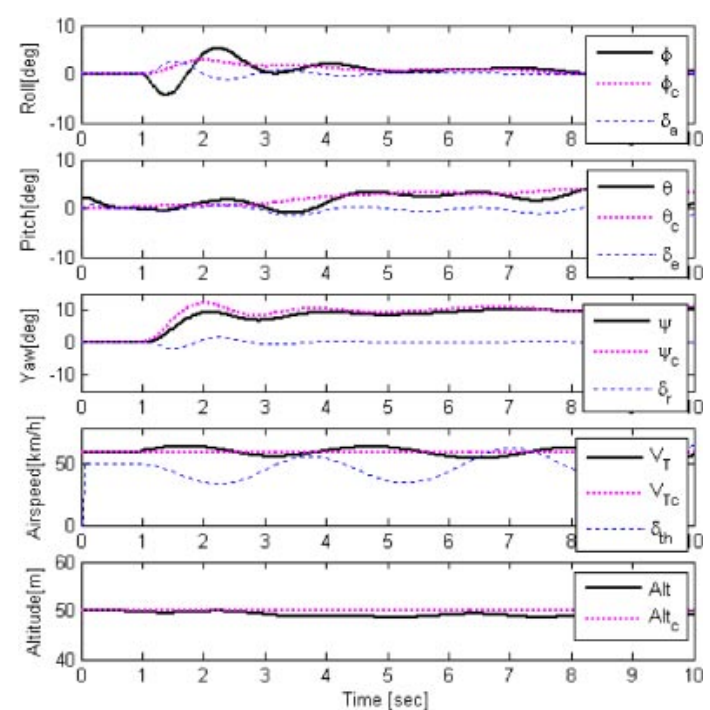

Fig. 8. Autopilot characteristics (case 2)
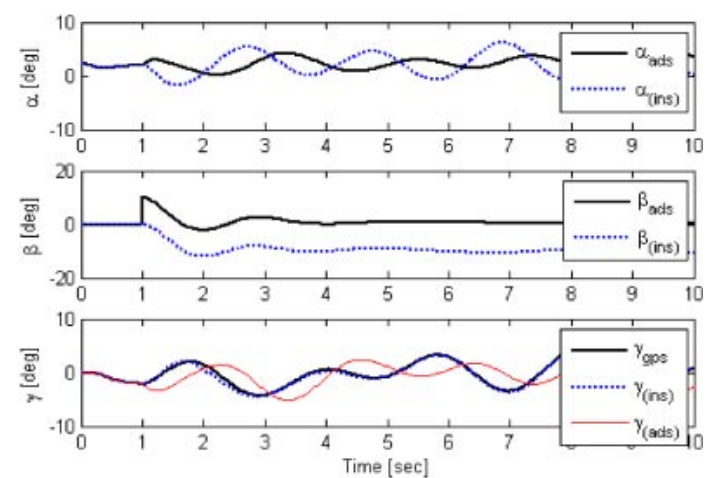

Fig. 10. Flight parameters (case 2).

Table 3. Approximation of $\alpha, \beta$ and $\gamma$.

\begin{tabular}{clc}
\hline Approximation & Wind excluded & Wind included \\
\hline$\alpha_{(I N S)}=\tan ^{-1} \frac{w_{\text {ins }}}{u_{\text {ins }}}$ & $\alpha_{\text {True }}=\alpha_{(I N S)}$ & $\alpha_{\text {True }} \neq \alpha_{(I N S)}$ \\
\hline$\beta_{(I N S)}=\tan ^{-1} \frac{v_{\text {ins }}}{u_{\text {ins }}}$ & $\beta_{\text {True }}=\beta_{(I N S)}$ & $\beta_{\text {True }} \neq \beta_{(I N S)}$ \\
\hline$\gamma_{(A D S)}=\theta_{\text {INS }}-\alpha_{A D S}$ & $\gamma_{\text {True }}=\gamma_{(A D S)}$ & $\gamma_{\text {True }} \neq \gamma_{(A D S)}$ \\
$\gamma_{(I N S)}=\theta_{\text {INS }}-\alpha_{I N S}$ & $\gamma_{\text {True }}=\gamma_{(I N S)}$ & $\gamma_{\text {True }} \cong \gamma_{(I N S)}$ \\
\hline$\alpha_{\text {True }}=\alpha_{A D S}, \beta_{\text {True }}=\beta_{\text {ADS }}, \gamma_{\text {True }}=\gamma_{G P S}$. &
\end{tabular}

$\alpha_{\text {True }}=\alpha_{A D S}, \beta_{\text {True }}=\beta_{A D S}, \gamma_{\text {True }}=\gamma_{G P S}$.

the wind correlation simulation works correctly.

It is observed in both cases that wind measurement was correctly conducted and that $\beta$ ( $\left.\beta_{A D S}\right)$ converged to 0 under steady crosswind conditions.

In a sense, $\alpha$ and $\beta$ sensors are equivalent to an anemoscope. The simulation discussed in this study showed that a UAV can perform accurate airborne wind measurement when it is equipped with both $\alpha$ and $\beta$ sensors, and ADS and GPS/INS sensors.

The wind measurement simulation presented in this study will be valuable for future research on UAV flight. 
Trans. Japan Soc. Aero. Space Sci., Vol. 58, No. 4, 2015

\section{References}

1) Choi, H. S., Lee, S., Lee, J., Kim, E. T., and Shim, H.: Aircraft Longitudinal Auto-landing Guidance Law Using Time Delay Control Scheme, T. Jpn. Soc. Aeronaut. Space Sci., 53 (2010), pp. 207-214.

2) Yoon, S. and Kim, Y.: Constrained Adaptive Backstepping Controller Design for Aircraft Landing in Wind Disturbance and Actuator Stuck, Int. J. Aeronaut. Space Sci., 13 (2012), pp. 74-89.

3) Allen, M. and Lin, V.: Guidance and Control of an Autonomous Soaring UAV, NASA/TM-2007-214611, 2007.

4) Bjarke, L. J. and Ehernberger, L. J.: An In-Flight Technique for Wind Measurement in Support of the Space Shuttle Program, NASA TM 4154, 1989, pp. 3-4.

5) Axford, D. N.: On the Accuracy of Wind Measurements Using an Inertial Platform in an Aircraft, and an Example of a Measurement of the
Vertical Mesostructure of the Atmosphere, J. Appl. Meteorol., 7 (1968), pp. 645-666.

6) Langelaan, J. W., Alley, N., and Neidhoefer, J.: Wind Field Estimation for Small Unmanned Aerial Vehicles, AIAA Guidance, Navigation and Control Conference, 2010.

7) Kroonenberg, A., Martin, T., Buschmann, M., Bange, J., and Vorsmann, P.: Measuring the Wind Vector Using the Autonomous Mini Aerial Vehicle $\mathrm{M}^{2}$ AV, Am. Meteorol. Soc., 25 (2008), pp. 1969-1982.

8) Cho, A., Kim, J., Lee, S., and Kee, C.: Wind Estimation and Airspeed Calibration using a UAV with a Single-Antenna GPS Receiver and Pitot Tube, IEEE Trans. Aerospace Electron. Syst., 47 (2011), pp. 109-115.

9) Lee, S., Lee, J., and Lee, D. S.: Lateral and Directional SCAS Controller Design Using Multidisciplinary Optimization Program, J. Korean Soc. Aeronaut. Space Sci., 40, 3 (2012), pp. 251-257 (in Korean). 